\title{
Updated Overall Survival in a Randomized Study Comparing Dacomitinib with Gefitinib as First-Line Treatment in Patients with Advanced Non-Small-Cell Lung Cancer and EGFR-Activating Mutations
}

\author{
Tony S. Mok ${ }^{1}$ Ying Cheng ${ }^{2} \cdot$ Xiangdong Zhou ${ }^{3} \cdot \mathrm{Ki} \mathrm{Hyeong} \mathrm{Lee}^{4} \cdot \mathrm{Kazuhiko} \mathrm{Nakagawa}^{5} \cdot$ Seiji Niho ${ }^{6} \cdot$ Alka Chawla $^{7}$. \\ Rafael Rosell ${ }^{8}$. Jesus Corral ${ }^{9} \cdot$ Maria Rita Migliorino $^{10} \cdot$ Adam Pluzanski $^{11} \cdot$ Kay Noonan $^{12} \cdot$ Yiyun Tang $^{13}$. \\ Malaika Pastel ${ }^{14} \cdot$ Keith D. Wilner $^{13} \cdot$ Yi-Long Wu ${ }^{15}$
}

Published online: 17 December 2020

(c) The Author(s) 2020

\begin{abstract} cant improvement in overall survival (OS) with dacomitinib. and alanine aminotransferase increased $(40.2 \%)$ with gefitinib. up in patients with advanced NSCLC with EGFR-activating mutations.

ClinicalTrials.gov NCT01774721 (registered 24 January 2013).

\section{Introduction}

The first-generation, reversible, epidermal growth factor receptor $(E G F R)$ tyrosine kinase inhibitors (TKIs) gefitinib and erlotinib improve progression-free survival (PFS) when
\end{abstract}

Background ARCHER 1050, an ongoing, randomized, open-label, phase III trial of dacomitinib versus gefitinib in newly diagnosed patients with advanced non-small-cell lung cancer (NSCLC) and an EGFR-activating mutation, reported signifi-

Objective This paper reports an updated OS analysis of ARCHER 1050 after an extended follow-up.

Patients and methods In this multinational, multicenter trial, adults (aged $\geq 18$ years or $\geq 20$ years in Japan and Korea) with newly diagnosed NSCLC and EGFR mutation (exon 19 deletion or exon 21 L858R substitution), and no history of central nervous system metastases, were randomized $1: 1$ to receive dacomitinib $45 \mathrm{mg} / \mathrm{day}(n=227)$ or gefitinib $250 \mathrm{mg} /$ day $(n=225)$. Randomization was stratified by race and EGFR mutation type. An ad hoc updated analysis of OS was conducted at the protocol-defined cut-off of 48 months from first dosing of the last enrolled patient (13 May 2019).

Results After a median follow-up of 47.9 months, 133 (58.6\%) patients had died in the dacomitinib arm and $152(67.6 \%)$ in the gefitinib arm. The hazard ratio (HR) for OS was 0.748 (95\% CI 0.591-0.947; two-sided $P=0.0155$ ); median OS was 34.1 months with dacomitinib versus 27.0 months with gefitinib. The HR for OS in patients with dose reduction(s) in the dacomitinib arm $(n=154)$ compared with all patients in the gefitinib arm was 0.554 (95\% CI 0.420-0.730); median OS was 42.5 months for patients with dose reduction(s) in the dacomitinib arm. The most common adverse events were diarrhea $(87.7 \%)$, paronychia (61.7\%), dermatitis acneiform (49.3\%), and stomatitis (43.6\%) with dacomitinib, and diarrhea (55.8\%)

Conclusions The OS benefit from first-line treatment with dacomitinib versus gefitinib was maintained after extended follow-

Electronic supplementary material The online version of this article (https://doi.org/10.1007/s40265-020-01441-6) contains supplementary material, which is available to authorized users.

Yi-Long Wu

syylwu@live.cn

Extended author information available on the last page of the article compared with chemotherapy but have not been shown to improve overall survival (OS) as a first-line treatment in patients with advanced non-small-cell lung cancer (NSCLC) harboring EGFR mutations [1-7]. The phase III ARCHER 1050 trial evaluated the efficacy and safety of a secondgeneration irreversible pan-HER TKI, dacomitinib, versus gefitinib in treatment-naïve patients with advanced NSCLC and an EGFR-activating mutation (exon 19 deletion or exon 21 L858R substitution) [8]. The primary analysis showed that dacomitinib was superior to gefitinib with respect to PFS as determined by a blinded independent radiologic central (BIRC) review (hazard ratio [HR]: 0.59; 95\% confidence interval (CI): 0.47-0.74; $P<0.0001)$; median PFS 


\section{Key Points}

This updated analysis confirmed improvement of overall survival with dacomitinib compared with gefitinib in patients with EGFR mutation after a median follow-up of 48 months.

The overall survival benefit from treatment with dacomitinib was maintained in patients who had a dose reduction.

Improvement of overall survival was observed in most of the predefined subgroups, including the Asian population and those with exon 21 L858R substitution mutation.

was 14.7 (95\% CI 11.1-16.6) versus 9.2 (95\% CI 9.1-11.0) months. TKI-associated adverse events (AEs) were generally managed by dose modifications and supportive care [9]. Although dose reductions due to AEs occurred in 150 (66\%) patients in the dacomitinib arm, permanent discontinuation due to dacomitinib-related AEs occurred in $22(10 \%)$ of patients [9]. Previous findings from the prespecified final analysis for OS in ARCHER 1050 (data cut-off on 17 February 2017) showed a significant improvement in OS with dacomitinib compared with gefitinib (HR 0.760; 95\% CI 0.582-0.993; two-sided $P=0.044$ ), with a median OS of 34.1 (95\% CI 29.5-37.7) versus 26.8 (95\% CI 23.7-32.1), after a median follow-up of 31.3 months [10]. Forty-nine (21.6\%) patients were still receiving dacomitinib at the time of the data cut-off for the prespecified final analysis. This was the first randomized, head-to-head comparison of two EGFR TKIs to show a significant improvement in OS, thus it is important to validate this observation with a longer follow-up and assess the impact of dacomitinib dose reduction on long-term survival. This paper reports an updated ad hoc OS analysis of ARCHER 1050 after an extended median follow-up of 47.9 months.

\section{Methods}

\subsection{Study Design, Patients, and Treatment}

Details of the ARCHER 1050 trial (ClinicalTrials.gov identifier: NCT01774721) have been reported [8]. Briefly, this is an ongoing, randomized, open-label, phase III trial in patients with newly diagnosed, stage IIIB/IV or recurrent NSCLC with tumor tissue testing positive for an EGFRactivating mutation (any exon 19 deletion or exon 21 L858R substitution). Patients who had received prior systemic therapy for locally advanced or metastatic NSCLC or who had any history or evidence of brain or leptomeningeal metastases or of diffuse non-infectious pneumonitis or interstitial lung disease were excluded. Patients were randomized (1:1) to receive dacomitinib $45 \mathrm{mg} /$ day or gefitinib $250 \mathrm{mg} /$ day in 28-day cycles. The randomization was stratified by race (Japanese, Chinese, other East Asian, or non-Asian) and EGFR mutation status (exon 19 deletion or exon 21 L858R substitution mutation). The primary endpoint was PFS by BIRC review (per Response Evaluation Criteria in Solid Tumors version 1.1 criteria) and OS was a key secondary endpoint.

Dacomitinib dose reductions from $45 \mathrm{mg} /$ day down to $30 \mathrm{mg} /$ day and then, if needed, from $30 \mathrm{mg} /$ day down to $15 \mathrm{mg} /$ day were allowed to manage treatment-related toxicity not controlled by optimal supportive care or not tolerated due to symptoms or interference with normal daily activities regardless of severity. If a patient tolerated treatment at the reduced dose level, in the judgment of the investigator, the dose could be increased to the next dose level. If a patient could not tolerate treatment after dose reduction to $15 \mathrm{mg} /$ day, treatment was permanently discontinued. For Grade 3 or intolerable Grade 2 toxicity, treatment was interrupted and only resumed at the same or an attenuated dacomitinib dose level upon recovery to Grade 2 or baseline, and in the clinical judgment of the investigator with agreement of the patient. For Grade 4 toxicity, treatment was interrupted and only resumed at the next lower dacomitinib dose level upon recovery to Grade 2 or baseline. The dose of gefitinib could be reduced from $250 \mathrm{mg}$ daily to every-other-day $250 \mathrm{mg}$ dosing following interruption due to Grade 4, Grade 3, or intolerable Grade 2 toxicity. If the patient tolerated the dose at every-other-day dosing, the investigator could return the patient to every day dosing. If the patient did not recover within 2 weeks of interruption for a drug-related AE, treatment was permanently discontinued unless agreement between the investigator and the sponsor, in which case treatment could resume.

The study was conducted in accordance with legal and regulatory requirements, the general principles in the International Ethical Guidelines for Biomedical Research Involving Human Subjects (Council for International Organizations of Medical Sciences 2002), and the Declaration of Helsinki (World Medical Association 1996 and 2008). Each patient provided written informed consent.

\subsection{Assessments}

As per the protocol, all patients were followed up for subsequent cancer therapies and survival status for up to 48 months from the date of first dosing of the last enrolled patient (13 May 2019). AEs were graded for severity according to the National Cancer Institute Common Terminology Criteria for Adverse Events, version 4.0, and assessed by the investigator for causality (data cut-off date: 13 May 2019). 


\subsection{Statistical Analyses}

The sample size was based on detecting a 50\% improvement in PFS by BIRC review (the primary endpoint). A gate-keeping procedure was used for hypotheses testing in a hierarchical approach to control the family-wise error rate for the analyses of the primary endpoint, and secondary endpoints of objective response rate (ORR) per BIRC review and OS in sequential order. Because the ORR did not reach statistical significance, a formal assessment of statistical benefit of OS could not be performed. Testing began with comparing PFS per BIRC review between the dacomitinib arm and the gefitinib arm with a one-sided significance level of 0.025 . As the null hypothesis for PFS was rejected, ORR per BIRC review was tested with a one-sided significance level of 0.025. Although the formal comparison of ORR was not statistically significant and the null hypothesis for ORR was not rejected, an ad hoc analysis of OS was conducted. Median OS was estimated using Kaplan-Meier (KM) methods, with two-sided 95\% CIs calculated. Stratified and unstratified logrank tests were used to assess the difference in OS between the two treatment arms. The HR and 95\% CI were estimated using the Cox proportional hazards model (adjusted for stratification factors). OS at 42 months (a post hoc exploratory endpoint) was defined as the probability of a patient being alive at 42 months from the date of randomization and was estimated using KM methods with a two-sided 95\% CI. Subgroup analyses of OS were conducted as supportive analyses, including the post hoc analyses in the dacomitinib arm by dose reduction levels (those whose dose remained at $45 \mathrm{mg} /$ day, and those whose dose reduced to $30 \mathrm{mg}$ /day or who then reduced from 30 to $15 \mathrm{mg} /$ day). OS for patients in the dacomitinib arm who received dose reduction was compared against that of all patients in the gefitinib arm (since dacomitinib was managed by dose reductions of the daily dose whereas gefitinib was managed by dosing every other day). Safety results were not adjusted for differing median treatment duration in the dacomitinib and gefitinib arms.

\section{Results}

\subsection{Patients and Treatment}

A total of 452 patients were randomized; 227 patients received dacomitinib and 225 patients received gefitinib (Online Resource 1). Demographics were generally similar between treatment arms but there were slightly more men in the gefitinib arm (44.4\%) than in the dacomitinib arm (35.7\%) (Online Resource 2). At the extended data cutoff date of 13 May 2019, 11 (5\%) patients on dacomitinib were ongoing (none remained in the gefitinib arm) (Online Resource 1). Median duration of treatment was 15.4 months (range: 0.07-60.5) in the dacomitinib arm and 12.0 months (range: 0.07-48.1) in the gefitinib arm.

\subsection{Overall Survival}

At the time of the updated OS analysis, 285 (63.1\%) patients had died (133 [58.6\%] in the dacomitinib arm and 152 [67.6\%] in the gefitinib arm) after a median follow-up of 47.9 months. OS was significantly longer in the dacomitinib arm than in the gefitinib arm (HR: 0.748; 95\% CI 0.591-0.947; two-sided $P=0.0155$ ); median OS was 34.1 months (95\% CI 29.5-39.8) versus 27.0 months (95\% CI 24.4-31.6) (Fig. 1). Despite the early crossing in the KM OS curves, the OS probability at 12 months from randomization was similar between the dacomitinib arm and the gefitinib arm (85.7\% (95\% CI 80.4-89.7) vs. $86.0 \%$ (95\% CI $80.7-89.9)$, respectively). OS probability at 42 months was $41.0 \%$ (95\% CI 34.3-47.6) in the dacomitinib arm and 33.6\% (95\% CI 27.2-40.0) in the gefitinib arm. Symptomatic brain metastases developed in one patient in the dacomitinib arm and nine patients in the gefitinib arm.

Subgroup analyses of OS by baseline characteristics were consistent with OS in the whole population (Fig. 2). For the subgroup of Asian patients (170/227 in the dacomitinib arm; $176 / 225$ in the gefitinib arm) in which $60.7 \%$ of patients had died across both arms, the HR for OS with dacomitinib versus gefitinib was 0.759 (95\% CI 0.578-0.996; two-sided $P=0-0457$ ), and the median OS was 37.7 months (95\% CI 30.2-44.7 months) versus 29.1 months (95\% CI 25.6-36.0) (Fig. 2 and Online Resource 3). For the subgroup of nonAsian patients (57/227 in the dacomitinib arm; 49/225 in the gefitinib arm) in which $70.8 \%$ of patients had died across both arms, the HR for OS with dacomitinib versus gefitinib was 0.758 (95\% CI $0.480-1.196$; two-sided $P=0.2318$; median 29.5 months [95\% CI 20.7-34.7] vs. 20.6 months [95\% CI 16.1-25.8]) (Fig. 2 and Online Resource 3). For the subgroup with the exon 19 deletion mutation (134/227 in the dacomitinib arm; 133/225 in the gefitinib arm), in which $41.9 \%$ of patients were censored, the HR for OS with dacomitinib versus gefitinib was 0.847 (95\% CI 0.618-1.161; two-sided $P=0.3021$ ), and the median OS was 36.7 months (95\% CI 30.2-44.3) versus 30.8 months (95\% CI 25.2-42.2) (Fig. 2 and Online Resource 4). For the subgroup with exon 21 L858R substitution mutation (93/227 in the dacomitinib arm; 92/225 in the gefitinib arm), in which $29.7 \%$ of patients were censored across both arms, the HR for OS with dacomitinib versus gefitinib was 0.665 (95\% CI $0.470-0.941$; two-sided $P=0.0203$ ), and the median OS was 32.5 months (95\% CI 25.5-39.5) versus 23.2 months (95\% CI 19.6-28.9) (Fig. 2 and Online Resource 4).

The HR for OS in the dacomitinib arm with dose reduction(s) $(n=154)$ compared with the gefitinib arm was 0.554 (95\% CI 0.420-0.730), and the median OS was 
Fig. 1 Updated overall survival (ITT). CI confidence interval, $H R$ hazard ratio, ITT intent-totreat, $O S$ overall survival, $O S_{42}$ overall survival probability at 42 months

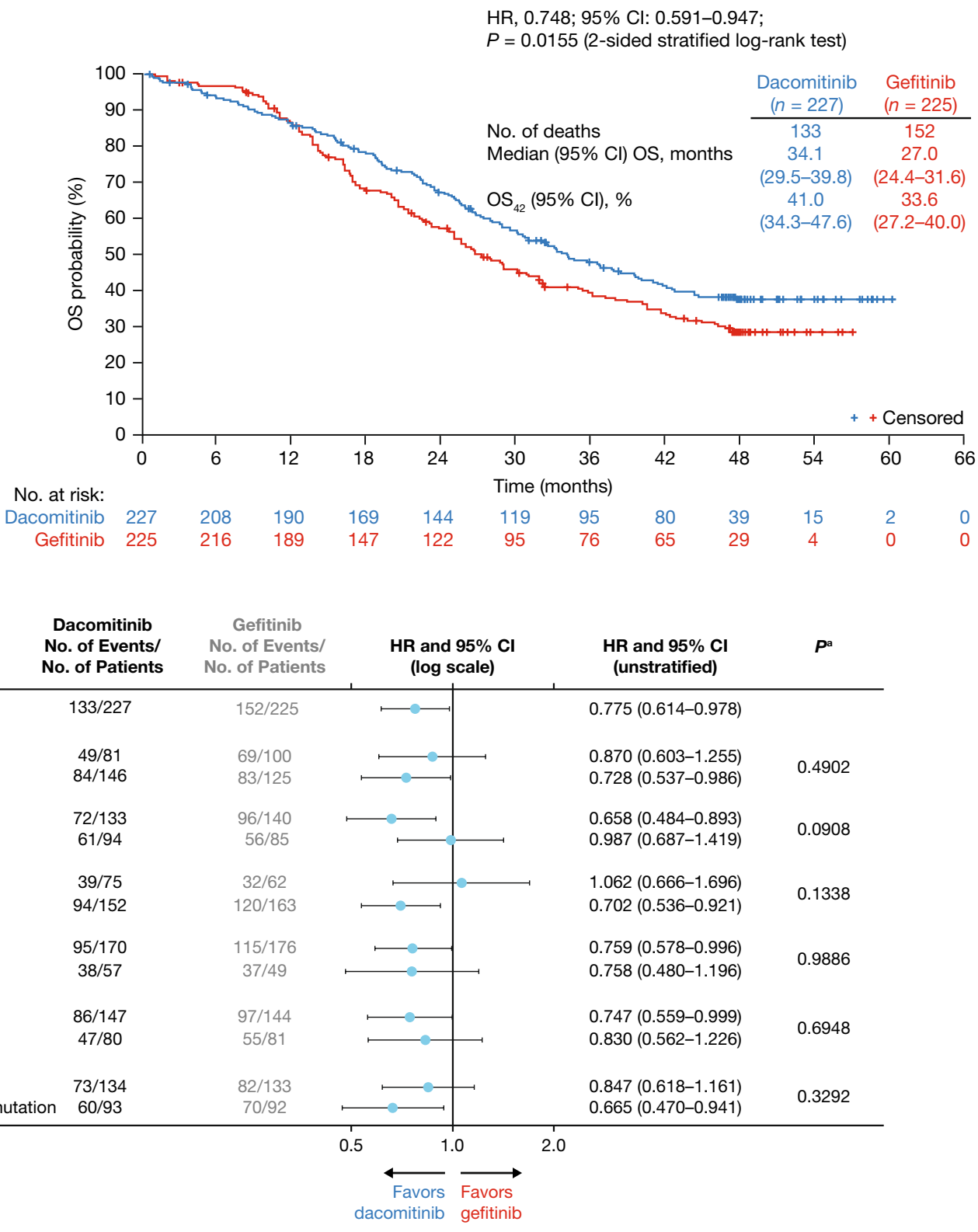

Fig. 2 Subgroup analyses for overall survival (ITT). ${ }^{\text {a }}$ Test for interaction (Wald Chi-Square test, two-sided). CI confidence interval, ECOG Eastern Cooperative Oncology Group, EGFR epidermal

42.5 months (95\% CI 36.7-not recorded [NR]) for dacomitinib with dose reduction (Fig. 3a) versus 27.0 months for gefitinib (95\% CI 24.4-31.6) (Fig. 1). Median OS was 20.7 months (95\% CI 15.4-25.6) in the subgroup of patients $(n=73)$ who had no dose reduction and remained on $45 \mathrm{mg} /$ day dacomitinib, 36.7 months (95\% CI 28.8-44.7 months) in patients $(n=89)$ whose dose was reduced to $30 \mathrm{mg} /$ day and not reached $(95 \%$ CI $40.1-\mathrm{NR})$ in patients $(n=65)$ whose dose was reduced from $45 \mathrm{mg} /$ day to $30 \mathrm{mg} /$ day to $15 \mathrm{mg} /$ day (Fig. 3b). However, these results should be interpreted cautiously since dose reduction was a study outcome (see growth factor receptor, $H R$ hazard ratio, ITT intent-to-treat, $O S$ overall survival, $P S$ performance status

Discussion). The median (range) treatment duration across dose levels received was 33.3 weeks $(0.3-259.0)$ for patients who remained on $45 \mathrm{mg} / \mathrm{day}, 64.0$ weeks (5.1-262.1) for patients whose lowest dose was $30 \mathrm{mg} /$ day, and 96.0 weeks (11.9-262.1) for patients whose lowest dose was $15 \mathrm{mg} /$ day.

\subsection{Subsequent Therapies}

Subsequent systemic therapies were received by 130 (57.3\%) patients in the dacomitinib arm and $146(64.9 \%)$ in the gefitinib arm. The most common subsequent systemic 
a
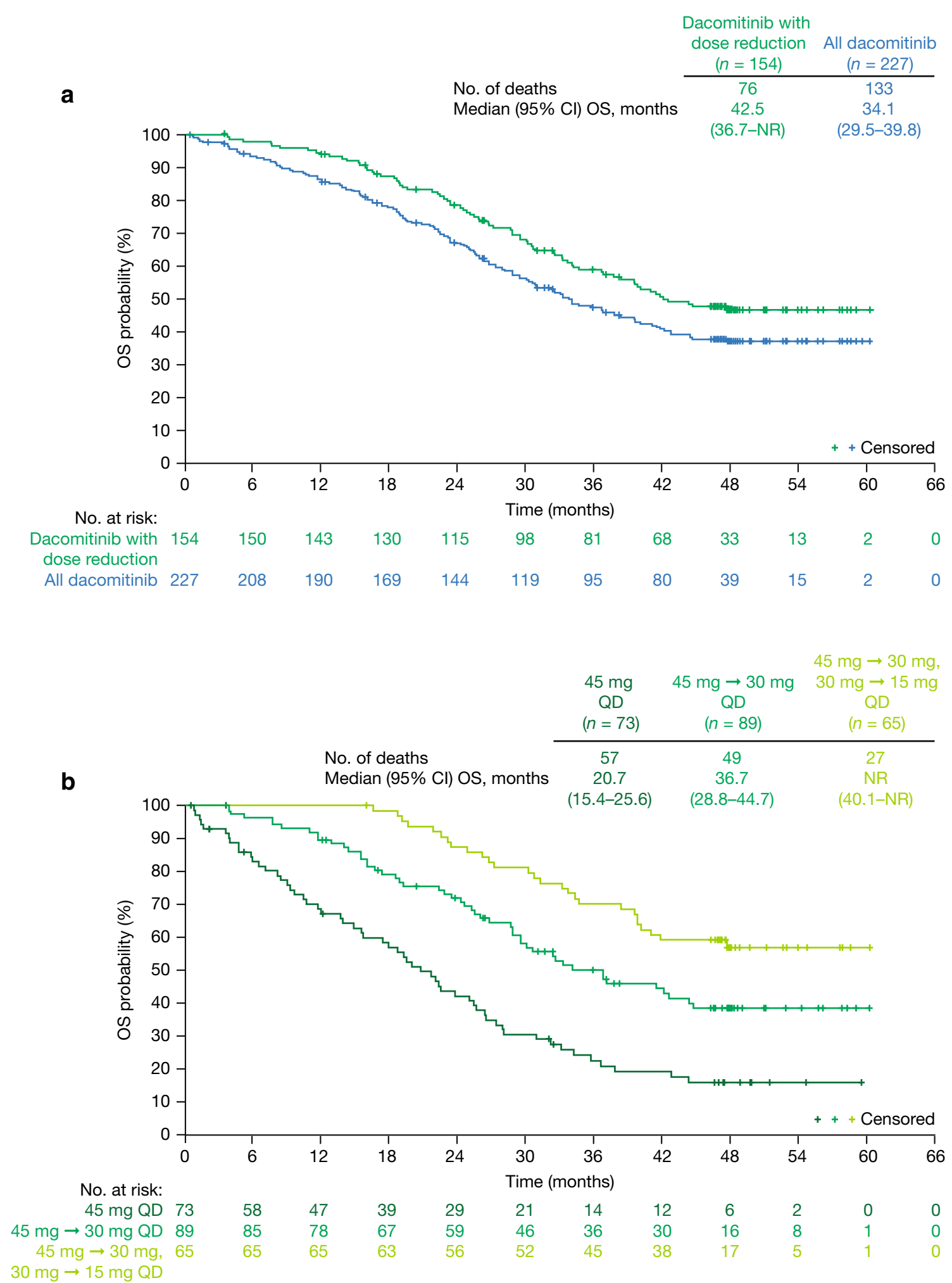

Fig. 3 Overall survival for (a) dose-reduced patients treated with dacomitinib and all dacomitinib-treated patients and (b) by lowest dose level of dacomitinib (ITT). ITT intent-to-treat, $O S$ overall survival 
therapies ( $\geq 10 \%$ of patients in either arm) were pemetrexed, osimertinib, cisplatin, carboplatin, docetaxel, gefitinib, and erlotinib (Online Resource 5). Third-generation EGFR TKIs were used as a first subsequent therapy in $32(14.1 \%)$ patients in the dacomitinib arm and $27(12.0 \%)$ patients in the gefitinib arm; the HR for OS for dacomitinib versus gefitinib in these patients was 0.911 (95\% CI 0.439-1.891); median 44.7 months (95\% CI 37.8-NR) versus 46.8 months (95\% CI 30.2-NR).

\subsection{Safety}

At the time of this updated analysis of OS, AEs were consistent with those reported in the primary analysis [8]. A summary of AEs is shown in Online Resource 6. The most frequently reported AEs of any grade in patients who received dacomitinib were diarrhea (199 [87.7\%] patients), paronychia (140 [61.7\%]), dermatitis acneiform (112 [49.3\%]), and stomatitis (99 [43.6\%]) (Table 1). The most frequently reported AEs of any grade in patients who received gefitinib were diarrhea (125 [55.8\%]), alanine aminotransferase (ALT) increased (90 [40.2\%]) and aspartate aminotransferase (AST) increased (84 [37.5\%]). The most frequently reported grade 3 or 4 AEs were dermatitis acneiform (31 [13.7\%]), diarrhea (19 [8.4\%]), and paronychia (18 [7.9\%]) in patients who received dacomitinib, and ALT increased (20 [8.9\%]) and AST increased (11 [4.9\%]) in patients who received gefitinib (Table 1). Serious AEs (SAEs) were reported by $69(30.4 \%)$ patients treated with dacomitinib and 53 (23.7\%) patients treated with gefitinib, and treatment-related SAEs by $22(9.7 \%)$ and $10(4.5 \%)$ patients, respectively. Treatment-related SAEs were most frequently due to diarrhea $(n=5)$, abdominal pain $(n=2)$, liver injury $(n=2)$, and decreased appetite $(n=2)$ in the dacomitinib arm and hepatic enzyme increased $(n=2)$ in the gefitinib arm. Deaths reported as AEs occurred in 24 (10.6\%) patients in the dacomitinib arm and $22(9.8 \%)$ patients in the gefitinib arm. These were most frequently

Table 1 All causality treat-emergent adverse events in $\geq 20 \%$ of patients (any grade) and/or $\geq 2 \%$ grade 3 or 4 (safety population)

\begin{tabular}{|c|c|c|c|c|c|c|}
\hline & \multicolumn{3}{|c|}{ Dacomitinib $(n=227)$} & \multicolumn{3}{|c|}{ Gefitinib $(n=224)$} \\
\hline & Any grade & Grade 3 & Grade 4 & Any grade & Grade 3 & Grade 4 \\
\hline Diarrhea & $199(87.7)$ & $19(8.4)$ & 0 & $125(55.8)$ & $2(0.9)$ & 0 \\
\hline Paronychia & $140(61.7)$ & $18(7.9)$ & 0 & $45(20.1)$ & $3(1.3)$ & 0 \\
\hline Dermatitis acneiform & $112(49.3)$ & $31(13.7)$ & 0 & $64(28.6)$ & 0 & 0 \\
\hline Stomatitis & 99 (43.6) & $8(3.5)$ & 0 & $41(18.3)$ & $1(0.4)$ & 0 \\
\hline Decreased appetite & $74(32.6)$ & $8(3.5)$ & 0 & $57(25.4)$ & $2(0.9)$ & 0 \\
\hline Weight decreased & $67(29.5)$ & $7(3.1)$ & 0 & $43(19.2)$ & $1(0.4)$ & 0 \\
\hline Dry skin & $64(28.2)$ & $3(1.3)$ & 0 & $39(17.4)$ & 0 & 0 \\
\hline ALT increased & $53(23.3)$ & $2(0.9)$ & 0 & $90(40.2)$ & $19(8.5)$ & $1(0.4)$ \\
\hline Alopecia & $53(23.3)$ & 0 & 0 & $29(12.9)$ & 0 & 0 \\
\hline AST increased & 49 (21.6) & $1(0.4)$ & 0 & $84(37.5)$ & $10(4.5)$ & $1(0.4)$ \\
\hline Cough & $47(20.7)$ & 0 & 0 & $45(20.1)$ & $2(0.9)$ & 0 \\
\hline Pruritus & $47(20.7)$ & $1(0.4)$ & 0 & $32(14.3)$ & $3(1.3)$ & 0 \\
\hline Conjunctivitis & $46(20.3)$ & 0 & 0 & $10(4.5)$ & 0 & 0 \\
\hline Rash & $41(18.1)$ & $10(4.4)$ & 0 & $26(11.6)$ & 0 & 0 \\
\hline Asthenia & $32(14.1)$ & $5(2.2)$ & 0 & $30(13.4)$ & $4(1.8)$ & 0 \\
\hline Dyspnea & $32(14.1)$ & $5(2.2)$ & $1(0.4)$ & $31(13.8)$ & $4(1.8)$ & 0 \\
\hline Rash maculopapular & $29(12.8)$ & $12(5.3)$ & 0 & $27(12.1)$ & $1(0.4)$ & 0 \\
\hline Dermatitis & $25(11.0)$ & $5(2.2)$ & 0 & $9(4.0)$ & $1(0.4)$ & 0 \\
\hline Hypokalemia & $25(11.0)$ & $10(4.4)$ & $2(0.9)$ & $13(5.8)$ & $4(1.8)$ & 0 \\
\hline Rash pustular & $15(6.6)$ & $8(3.5)$ & 0 & $3(1.3)$ & 0 & 0 \\
\hline Pleural effusion & $6(2.6)$ & $5(2.2)$ & 0 & $6(2.7)$ & $1(0.4)$ & 0 \\
\hline Lymphocyte count decreased & $5(2.2)$ & $5(2.2)$ & 0 & $3(1.3)$ & 0 & 0 \\
\hline Hypertension & $19(8.4)$ & $4(1.8)$ & 0 & $21(9.4)$ & $7(3.1)$ & 0 \\
\hline Anemia & $27(11.9)$ & $3(1.3)$ & 0 & $18(8.0)$ & $5(2.2)$ & 0 \\
\hline Hepatic function abnormal & $5(2.2)$ & $2(0.9)$ & 0 & $8(3.6)$ & $5(2.2)$ & 0 \\
\hline
\end{tabular}

$A L T$ alanine aminotransferase, $A S T$ aspartate aminotransferase 
due to disease progression $(n=9)$, pneumonia $(n=2)$, metastases to meninges $(n=2)$, respiratory failure $(n=2)$ in the dacomitinib arm, and disease progression $(n=12)$, dyspnea $(n=2)$, malignant neoplasm progression $(n=2)$ in the gefitinib arm. Permanent discontinuation associated with AEs related to study drug occurred in $23(10.1 \%)$ patients in the dacomitinib arm and $15(6.7 \%)$ in the gefitinib arm, and were most frequently due to dermatitis acneiform $(n=3)$, diarrhea $(n=2)$, interstitial lung disease $(n=2)$, stomatitis $(n=2)$, pneumonia $(n=2)$, and rash maculopapular $(n=2)$ in the dacomitinib arm, and increased ALT $(n=2)$, increased AST $(n=2)$, increased hepatic enzyme $(n=2)$, and pneumonitis $(n=2)$ in the gefitinib arm.

Dose reductions due to AEs occurred in 153 (67.4\%) patients in the dacomitinib arm and 18 (8.0\%) patients in the gefitinib arm. Among the dacomitinib-treated patients, dose reductions (any cause) occurred in 154 (67.8\%) patients (AE actions did not include dose reduction for one of these patients), while 73 (32.2\%) patients remained at $45 \mathrm{mg} /$ day for the duration of treatment. Of the 227 patients in the dacomitinib arm, 89 (39.2\%) patients reduced to $30 \mathrm{mg} /$ day as the lowest dose and $65(28.6 \%)$ patients reduced from $45 \mathrm{mg}$ /day to $30 \mathrm{mg} /$ day and then to $15 \mathrm{mg}$ /day as the lowest dose.

\section{Discussion}

The results of this updated ad hoc OS analysis after extended follow-up confirmed prior observation of improvement in OS with dacomitinib versus gefitinib as first-line treatment in patients with newly diagnosed, locally advanced or metastatic NSCLC with EGFR-activating mutations and without central nervous system (CNS) metastases, and are consistent with the prespecified final OS analysis [10]. The HR of 0.748 and improvement in median OS of 7 months with dacomitinib versus gefitinib are beyond the clinically meaningful targets for non-squamous cell carcinoma (OS improvement over 3.25-4 months and target HR below 0.76-0.8) as defined by an American Society of Clinical Oncology working group [11]. Further examination of the early crossing of the curves showed that this is likely due to random chance and is illustrative of how the timing of relatively small numbers of events can influence the shape of a KM plot early in a study. The separation of the KM curves of dacomitinib from gefitinib persisted after 12 months and the crossing of the KM curves observed at 36 months in the prespecified final analysis of OS (after 220 deaths) [10] is no longer observed with the extended follow-up and more mature survival data (285 deaths). Only one other trial has shown a significant improvement in OS over a first-generation EGFR TKI as first-line monotherapy in patients with advanced NSCLC with EGFR-activating mutations. In the FLAURA trial comparing patients treated with either osimertinib $(n=279)$ or a first-generation EGFR TKI (gefitinib or erlotinib) $(n=277)$, the HR for OS at the final analysis was 0.80 (95.05\% CI: $0.64-1.00 ; P=0.046$; median 38.6 months [ $95 \%$ CI $34.5-41.8$ ] vs. 31.8 months [95\% CI 26.6-36.0]) [12]. The LUX Lung-7 trial found no significant difference in OS between patients with EGFRmutated advanced NSCLC who received first-line treatment with the second-generation EGFR TKI afatinib $(n=160)$ compared with those who received gefitinib $(n=159)$ (HR: $0.86 ; 95 \%$ CI $0.66-1.12 ; P=0.2580$; median 27.9 months vs. 24.5 months) [13]. Notably, $16 \%$ of patients from this study had stable asymptomatic CNS metastases at baseline [14].

In this updated OS analysis of ARCHER 1050, significant improvement of OS with dacomitinib versus gefitinib was observed in the Asian subgroup and in patients with exon 21 L858R substitution mutation. Although in the FLAURA trial, the OS benefit with osimertinib versus gefitinib/erlotinib was consistent across most subgroups, including the exon 19 deletion subgroup, the magnitude of benefit varied; in the Asian subgroup the HR was 1.00 (95\% CI 0.75-1.32) and in the exon $21 \mathrm{~L} 858 \mathrm{R}$ substitution mutation subgroup the HR was 1.00 (95\% CI 0.71-1.40). In the LUX Lung-7 trial, the HR for OS with afatinib versus gefitinib in the Asian subgroup was 0.95 (95\% CI 0.67-1.33), and results in patients with exon 19 deletion (HR: 0.83 ; 95\% CI 0.58-1.17; $P=0.2841$; median 30.7 versus 26.4 months) and exon 21 L858R substitution mutation (HR: 0.91; 95\% CI 0.62-1.36; $P=0.6585$; median 25.0 vs. 21.2 months) were generally consistent with the overall study population [13].

No new safety signals were observed after extended follow-up. AEs experienced during treatment with dacomitinib were managed with dose modifications, with dose reductions occurring in two-thirds of patients in the dacomitinib group. As previously reported, global quality of life showed a statistically significant but not clinically meaningful improvement in the gefitinib group, and was maintained in the dacomitinib group, despite clinically meaningful increases in the symptoms of diarrhea and sore mouth [8]. Most dacomitinibrelated AEs can be effectively managed with dose modifications and standard medical management [15].

The OS benefit from dacomitinib treatment in patients who had a dose reduction was comparable to the effect of dacomitinib treatment in all patients. This agrees with the analysis of these data at an earlier cut-off date [9] in which comparable median PFS and OS data were obtained in all patients treated with dacomitinib and those with dose reduction(s). However, the OS results in patients who had a dose reduction or by lowest dose received, including those patients with no dose reduction, need to be interpreted cautiously since dose reduction was a study outcome; therefore, OS by these factors was not based on criteria present at randomization [9]. Nonresponding patients with a shorter 
treatment duration are less likely to have had a dose reduction; similarly, responding patients with a longer treatment duration are perhaps more likely to experience AEs and require a dose reduction. Median time to dose reduction for patients whose lowest dose was $30 \mathrm{mg} /$ day was 13 weeks [9]. For patients whose lowest dose was $15 \mathrm{mg} /$ day, median time to first dose reduction (to $30 \mathrm{mg} / \mathrm{day}$ ) was 8 weeks, followed by a subsequent median time to a second dose reduction (from $30 \mathrm{mg} /$ day to $15 \mathrm{mg} /$ day) of 12 weeks. None of the baseline characteristics appeared to be predictive of which patients would have a dose reduction; however, dose reductions were more common in females, and patients with dose reductions had a lower median body weight [9]. Pharmacokinetic analysis showed that patients who remained on $45 \mathrm{mg} /$ day tended to have lower initial dacomitinib exposure compared with those who reduced dose [9]. Based on the study design, since all patients initially received $45 \mathrm{mg} /$ day of dacomitinib, no conclusions can be made on the potential efficacy of starting doses of either $30 \mathrm{mg} /$ day or $15 \mathrm{mg} /$ day. An ongoing Phase 2 trial (ATORG-003) in Asian countries is evaluating an alternative dose titration strategy whereby patients will be administered $30 \mathrm{mg} /$ day dacomitinib for 4 weeks, after which those with $\leq$ Grade 1 dacomitinibrelated toxicity may escalate to $45 \mathrm{mg} / \mathrm{day}$ [16].

A limitation of this updated OS analysis is that it was an ad hoc analysis. The hierarchical statistical testing order was PFS followed by ORR and then OS. While the updated OS result was statistically significant when assessed on its own, since the gate-keeping procedure stopped at the testing of ORR (per BIRC review) as ORR was not statistically significant, the statistical significance of OS improvement could not be formally assessed. The emphasis in this analysis was therefore on estimation of treatment effect rather than hypothesis testing and the $P$-values should be viewed descriptively and interpreted with caution. OS may also be confounded by subsequent systemic anticancer therapies. Subsequent systemic therapies were received by 130 (57.3\%) patients in the dacomitinib arm and 146 (64.9\%) patients in the gefitinib arm, with third-generation EGFR TKIs received as the first subsequent therapy by only 32 (14.1\%) patients and $27(12.0 \%)$ patients in the dacomitinib and gefitinib group, respectively, due to their limited availability when the study was conducted. However, at the time of the prespecified final OS analysis, OS censored for first subsequent therapy was consistent with the overall result (with third-generation EGFR TKIs received as the first subsequent therapy in 22 [9.7\%] patients and 25 [11.1\%] patients, respectively) [10]. Further studies are needed to investigate the optimal sequence of EGFR TKIs, as either monotherapy or in a combination regimen, for the treatment of EGFR mutation-positive advanced NSCLC. The exclusion of patients with any mutation other than exon 19 deletion or exon 21 L858R substitution (including less common EGFR-activating mutations), with or without exon 20 T790M mutation, and the exclusion of patients with CNS metastases are additional limitations of the ARCHER 1050 trial. Data on the incidence of T790M mutation after progression or on treatment would also have been of interest. Analysis of plasma samples from the Phase 2 ARCHER 1017 study showed that of the 15 patients with detectable EGFR-activating mutations, eight (53\%) tested positive for T790M at the end of treatment [17].

In conclusion, the OS benefit from first-line treatment with dacomitinib versus gefitinib was maintained after extended follow-up in patients with advanced NSCLC with $E G F R$-activating mutations and persisted in patients who had a dose reduction. Improvement of OS was observed in most of the predefined subgroups, including the Asian population and those with exon 21 L858R substitution mutation.

Acknowledgements The authors would like to thank the study participants and study site personnel.

Medical writing support was provided by Annette Smith, $\mathrm{PhD}$, of CMC AFFINITY, McCann Health Medical Communications, and was funded by Pfizer Inc.

\section{Declarations}

Funding This study was sponsored by Pfizer Inc and SFJ Pharmaceuticals.

Conflict of interest Tony Mok: grant/research funding (paid to institution): AstraZeneca, Bristol-Myers Squibb, Clovis Oncology, G1 Therapeutics, Merck Sharp \& Dohme, Merck Serono, Novartis, Pfizer, Roche, SFJ, Takeda, and XCovery; speaker's fees: ACEA Pharma, Alpha Biopharma Co., Ltd., Amgen, Amoy Diagnostics Co., LTD., AstraZeneca (before Jan 2019), Boehringer Ingelheim, Bristol-Myers Squibb, Eli Lilly, InMed Medical Communication, Merck Sharp \& Dohme, Novartis, Pfizer, PrIME Oncology, Roche/Genentech, Taiho, and Takeda Oncology; honoraria for consultation services: Abbvie Inc., ACEA Pharma (since Aug 2018), Alpha Biopharma Co., Ltd., Amgen, Amoy Diagnostics Co., Ltd., AstraZeneca (before Jan 2019), Bayer, Boehringer Ingelheim, Blueprint Medicines Corporation, Bristol-Myers Squibb, Celgene, CStone Pharmaceuticals, Daiichi Sankyo, Eli Lilly, Fishawack Facilitate Ltd., Hengrui Therapeutics Inc., Ignyta, Inc., Incyte Corporation, InMed Medical Communication, IQVIA, Janssen, Loxo-Oncology, Merck Serono, Merck Sharp \& Dohme, MoreHealth, Novartis, OncoGenex Pharmaceuticals, Inc., OrigiMed, PeerVoice, Pfizer, PrIME Oncology, Roche/Genentech, Sanofi-Aventis R\&D, SFJ Pharmaceutical Ltd., Takeda Pharmaceuticals HK Ltd., Vertex Pharmaceuticals, Yuhan Corporation, Medscape/WebMD (medical education/CME activities), PeerVoice (independent medical education), Prime Oncology (medical education); stock shareholder/ option: Clearbridge Biomedics (now Biolidics Ltd.), Hutchison ChiMed, Loxo-Oncology, OrigiMed Co. Ltd., Sanomics Ltd., and Virtus Medical Group; advisory board: Abbvie Inc., ACEA Pharma, Amgen, AstraZeneca, Bayer, Blueprint Medicines Corporation, Boehringer Ingelheim, Bristol-Myers Squibb, Celgene, Cirina, CStone Pharmaceuticals, Daiichi Sankyo, Eli Lilly, Fishawack Facilitate Ltd., G1 Therapeutics, Inc., geneDecode Co., Ltd. (uncompensated), Hengrui Therapeutics Inc., Hutchison Chi-Med, Ignyta, Inc., Incyte Corporation, IQVIA, Janssen, Loxo-Oncology, Lunit, Inc., Merck Serono, Merck Sharp \& Dohme, Novartis, OncoGenex Technologies Inc., 
OrigiMed, Pfizer, Roche/Genentech, Sanofi-Aventis R\&D, SFJ Pharmaceutical, Takeda Oncology, Vertex Pharmaceuticals, Virtus Medical Group, and Yuhan Corporation; board of directors/leadership (remunerated): AstraZeneca and Hutchison Chi-Med; board of directors/ leadership (non-remunerated): American Society of Clinical Oncology (ASCO), Asian Thoracic Oncology Research Group (ATORG), Chinese Lung Cancer Research Foundation Limited (CLCRF), Chinese Society of Clinical Oncology (CSCO), Hong Kong Cancer Fund (HKCF), Hong Kong Cancer Therapy Society (HKCTS), International Association for the Study of Lung Cancer (IASLC; term ended Apr 2019), and St. Stephen's College \& Prep. School. Kazuhiko Nakagawa: grants: MSD K.K., A2 Healthcare Corp., inVentiv Health Japan Astellas Pharm Inc., Daiichi Sankyo Co., Ltd., Novarits Pharma K.K., AbbVie Inc., Quintiles Inc., IQVIA Services JAPAN K.K., ICON Japan K.K., Chugai Pharmaceutical Co., Ltd., Takeda Pharmaceutical Co., Ltd., EP-CRSU CO., LTD., GRITSONE ONCOLOGY.INC, Linical Co., Ltd., Eli Lilly Japan K.K., Eisai Co., Ltd., Bristol-Myers Squibb, Nippon Boehringer Ingelheim Co., Ltd., Taiho Pharmaceutical Co., Ltd., Pfizer Japan Onc., PAREXEL International Corp., SymBio Pharmaceuticals Limited, Ono Pharmacuetical Co., Ltd., Kyowa Hakko Kirin Co., Ltd., EPS Corporation, Bayer Yakuhin Ltd., Syneos Health, EPS International Co., Ltd., Pfizer R\&D Japan G.K., Otsuka Pharmaceutical Co., Ltd.; honoraria: AstraZeneca K.K., Nichi-Iko Pharmaceutical Co., Ltd., Astellas Pharma Inc., Takeda Pharmaceutical Co., Ltd., MSD K.K., Taiho Pharmaceutical Co., Ltd., Ono Pharmaceutical Co., Ltd., Bristol-Myers Squibb, Nippon Boehringer Ingelheim Co., Ltd., Eli Lilly Japan K.K., Novartis Pharma K.K., SymBio Pharmaceuticals Limited, Pfizer Japan Inc., Chugai Pharmaceutical Co., Ltd., Clinical Trial Co., Ltd., NANZANDO Co., Ltd., MEDICUS SHUPPAN Publishers Co., Ltd., YODOSHA CO., LTD., Care Net Inc., Nikkei Business Publications Inc., Reno Medical K.K., Daiichi Sankyo Co., Ltd., KYORIN Pharmaceutical Co., Ltd., Thermo Fisher Scientific K.K., Medical Review Co., Ltd., YOMIURI TELECASTING CORPORATION, Roche Diagnostics K.K., Nippon Kayaku Co., Ltd., Bayer Yakuhin Ltd., Merck Biopharma Co., Ltd., Medical Mobile Communications Co., Ltd., AbbVie Inc., 3H Clinical Trial Inc. Seiji Niho: grants: AstraZeneca, Pfizer, Merck Biopharma, Eli Lilly, MSD, and Chugai; honoraria: AstraZeneca, Pfizer, Eli Lilly, Chugai, Taiho, MSD, Bristol-Myers Squibb, Novartis, Boehringer Ingelheim, Taiho, Ono, and Shionogi. Alka Chawla: former employee of SFJ Pharmaceuticals. Jesus Corral: Education grants, provided consultation, attended advisory boards and/or provided lectures for Pfizer, AstraZeneca, Roche, Eli Lilly, MSD, and Bristol-Myers Squibb. Adam Pluzanski: lecture fees and travel grants: Pfizer. Kay Noonan, Yiyun Tang, Malaika Pastel, and Keith Wilner: Pfizer employees and stockholders. Yi-Long Wu: grants (to institute): AstraZeneca, BMS, Boehringer Ingelheim, Pfizer, and Roche; honoraria: AstraZeneca, Boehringer Ingelheim, MSD, and Roche; lectures/speakers bureaus: AstraZeneca, BMS, Boehringer Ingelheim, Eli Lilly, MSD, Pfizer, and Roche. Ying Cheng, Xiangdong Zhou, Ki Hyeong Lee, Rafael Rosell, and Maria Rita Migliorino: no conflicts of interest to declare.

Ethical approval The study was conducted in accordance with legal and regulatory requirements, the general principles in the International Ethical Guidelines for Biomedical Research Involving Human Subjects (Council for International Organizations of Medical Sciences 2002), and the Declaration of Helsinki (World Medical Association 1996 and 2008).

Informed consent Informed consent was obtained from all individual participants included in the study.

Availability of data and material Upon request, and subject to certain criteria, conditions and exceptions (see https://www.pfizer.com/scien ce/clinical-trials/trial-data-and-results for more information), Pfizer will provide access to individual de-identified participant data from
Pfizer-sponsored global interventional clinical studies conducted for medicines, vaccines and medical devices (1) for indications that have been approved in the US and/or EU or (2) in programs that have been terminated (i.e., development for all indications has been discontinued). Pfizer will also consider requests for the protocol, data dictionary, and statistical analysis plan. Data may be requested from Pfizer trials 24 months after study completion. The de-identified participant data will be made available to researchers whose proposals meet the research criteria and other conditions, and for which an exception does not apply, via a secure portal. To gain access, data requestors must enter into a data access agreement with Pfizer.

Code availability Not applicable.

Open Access This article is licensed under a Creative Commons Attribution-NonCommercial 4.0 International License, which permits any non-commercial use, sharing, adaptation, distribution and reproduction in any medium or format, as long as you give appropriate credit to the original author(s) and the source, provide a link to the Creative Commons licence, and indicate if changes were made. The images or other third party material in this article are included in the article's Creative Commons licence, unless indicated otherwise in a credit line to the material. If material is not included in the article's Creative Commons licence and your intended use is not permitted by statutory regulation or exceeds the permitted use, you will need to obtain permission directly from the copyright holder. To view a copy of this licence, visit http://creativecommons.org/licenses/by-nc/4.0/.

\section{References}

1. Maemondo M, Inoue A, Kobayashi K, Sugawara S, Oizumi S, Isobe $\mathrm{H}$, et al. Gefitinib or chemotherapy for non-small-cell lung cancer with mutated EGFR. N Engl J Med. 2010;362(25):2380-8.

2. Rosell R, Carcereny E, Gervais R, Vergnenegre A, Massuti B, Felip E, et al. Erlotinib versus standard chemotherapy as firstline treatment for European patients with advanced EGFR mutation-positive non-small-cell lung cancer (EURTAC): a multicentre, open-label, randomised phase 3 trial. Lancet Oncol. 2012;13(3):239-46.

3. Wu YL, Zhou C, Liam CK, Wu G, Liu X, Zhong Z, et al. First-line erlotinib versus gemcitabine/cisplatin in patients with advanced EGFR mutation-positive non-small-cell lung cancer: analyses from the phase III, randomized, open-label. ENSURE study. Ann Oncol. 2015;26(9):1883-9.

4. Zhou C, Wu YL, Chen G, Feng J, Liu XQ, Wang C, et al. Final overall survival results from a randomised, phase III study of erlotinib versus chemotherapy as first-line treatment of EGFR mutation-positive advanced non-small-cell lung cancer (OPTIMAL, CTONG-0802). Ann Oncol. 2015;26(9):1877-83.

5. Fukuoka M, Wu YL, Thongprasert S, Sunpaweravong P, Leong SS, Sriuranpong V, et al. Biomarker analyses and final overall survival results from a phase III, randomized, open-label, firstline study of gefitinib versus carboplatin/paclitaxel in clinically selected patients with advanced non-small-cell lung cancer in Asia (IPASS). J Clin Oncol. 2011;29(21):2866-74.

6. Inoue A, Kobayashi K, Maemondo M, Sugawara S, Oizumi S, Isobe $\mathrm{H}$, et al. Updated overall survival results from a randomized phase III trial comparing gefitinib with carboplatin-paclitaxel for chemo-naive non-small cell lung cancer with sensitive EGFR gene mutations (NEJ002). Ann Oncol. 2013;24(1):54-9.

7. Yoshioka H, Shimokawa M, Seto T, Morita S, Yatabe Y, Okamoto I, et al. Final overall survival results of WJTOG3405, a randomized phase III trial comparing gefitinib versus cisplatin with 
docetaxel as the first-line treatment for patients with stage IIIB/IV or postoperative recurrent EGFR mutation-positive non-small-cell lung cancer. Ann Oncol. 2019;30(12):1978-84.

8. Wu YL, Cheng Y, Zhou X, Lee KH, Nakagawa K, Niho S, et al. Dacomitinib versus gefitinib as first-line treatment for patients with EGFR-mutation-positive non-small-cell lung cancer (ARCHER 1050): a randomised, open-label, phase 3 trial. Lancet Oncol. 2017;18(11):1454-66.

9. Corral J, Mok TS, Nakagawa K, Rosell R, Lee KH, Migliorino $\mathrm{MR}$, et al. Effects of dose modifications on the safety and efficacy of dacomitinib for EGFR mutation-positive non-small-cell lung cancer. Future Oncol. 2019;15(24):2795-805.

10. Mok TS, Cheng Y, Zhou X, Lee KH, Nakagawa K, Niho S, et al. Improvement in overall survival in a randomized study that compared dacomitinib with gefitinib in patients with advanced nonsmall-cell lung cancer and EGFR-activating mutations. J Clin Oncol. 2018;36(22):2244-50.

11. Ellis LM, Bernstein DS, Voest EE, Berlin JD, Sargent D, Cortazar $\mathrm{P}$, et al. American Society of Clinical Oncology perspective: raising the bar for clinical trials by defining clinically meaningful outcomes. J Clin Oncol. 2014;32(12):1277-80.

12. Ramalingam SS, Vansteenkiste J, Planchard D, Cho BC, Gray JE, Ohe Y, et al. Overall survival with osimertinib in untreated, EGFR-mutated advanced NSCLC. N Engl J Med. 2020;382(1):41-50.
13. Paz-Ares L, Tan EH, O’Byrne K, Zhang L, Hirsh V, Boyer M, et al. Afatinib versus gefitinib in patients with EGFR mutation-positive advanced non-small-cell lung cancer: overall survival data from the phase IIb LUX-Lung 7 trial. Ann Oncol. 2017;28(2):270-7.

14. Park K, Tan EH, O’Byrne K, Zhang L, Boyer M, Mok T, et al. Afatinib versus gefitinib as first-line treatment of patients with EGFR mutation-positive non-small-cell lung cancer (LUX-Lung 7): a phase $2 \mathrm{~B}$, open-label, randomised controlled trial. Lancet Oncol. 2016;17(5):577-89.

15. Zhou Q, Wu YL, Corral J, Nakagawa K, Garon EB, Sbar EI, et al. Management of common adverse events related to first-line dacomitinib use in EGFR mutation-positive non-small-cell lung cancer: a pooled safety analysis. Future Oncol. 2019;15(13):1481-91.

16. Tan A, Mok T, Kim D, Yang JC, Lee C, Soo R, et al. ATORG-003: dacomitinib with or without dose titration as first-line therapy for metastatic EGFR mutant non-small cell lung cancer. J Thorac Oncol. 2019;14(10 (Supplement)):S836-7.

17. Janne PA, Ou SI, Kim DW, Oxnard GR, Martins R, Kris MG, et al. Dacomitinib as first-line treatment in patients with clinically or molecularly selected advanced non-small-cell lung cancer: a multicentre, open-label, phase 2 trial. Lancet Oncol. $2014 ; 15(13): 1433-41$.

\section{Affiliations}

Tony S. Mok ${ }^{1}$ Y Ying Cheng ${ }^{2} \cdot$ Xiangdong Zhou $^{3} \cdot \mathrm{Ki} \mathrm{Hyeong} \mathrm{Lee}^{4} \cdot \mathrm{Kazuhiko} \mathrm{Nakagawa}^{5} \cdot$ Seiji Niho $^{6} \cdot$ Alka Chawla $^{7}$. Rafael Rosell $^{8}$. Jesus Corral ${ }^{9} \cdot$ Maria Rita Migliorino $^{10} \cdot$ Adam Pluzanski $^{11} \cdot$ Kay Noonan $^{12} \cdot$ Yiyun Tang $^{13}$. Malaika Pastel ${ }^{14} \cdot$ Keith D. Wilner $^{13} \cdot$ Yi-Long Wu $^{15}$

1 State Key Laboratory of Translational Oncology, Department of Clinical Oncology, Chinese University of Hong Kong, Hong Kong, China

2 Jilin Provincial Cancer Hospital, Changchun, China

3 First Affiliated Hospital of Third Military Medical University, Chongqing, China

4 Chungbuk National University Hospital, Chungbuk National University College of Medicine, Cheongju, South Korea

5 Kindai University Hospital, Osaka, Japan

6 National Cancer Center Hospital East, Kashiwa, Japan

7 SFJ Pharmaceuticals ${ }^{\circledR}$, Pleasanton, CA, USA

8 Catalan Institute of Oncology, Barcelona, Spain

9 Hospital Universitario Virgen del Rocio, Seville, Spain
10 Pulmonary Oncology Unit, San Camillo-Forlanini Hospital, Rome, Italy

11 Maria Sklodowska-Curie National Research Institute of Oncology, Warsaw, Poland

12 Pfizer Inc., Groton, CT, USA

13 Pfizer Oncology, La Jolla, CA, USA

14 Pfizer Oncology, New York, NY, USA

15 Guangdong Lung Cancer Institute, Guangdong Provincial People's Hospital and Guangdong Academy of Medical Sciences, 106 Zhongshan Er Rd, Guangzhou, Guangdong 510080, China 